\title{
Aplicativo móvel para a práxis educativa de enfermeiros da Estratégia Saúde da Família: ideação e prototipagema
}

Mobile application for the educational praxis of nurses in the Family Health Strategy: ideation and prototyping

Aplicación móvil para la praxis educativa de enfermeros en la Estrategia de Salud Familiar: ideación y creación de protótipos

\author{
Darlisom Sousa Ferreira ${ }^{12}$ (D) \\ Flávia Regina Souza Ramos ${ }^{12}$ (1) \\ Elizabeth Teixeira ${ }^{1}$ (1)
}

1. Universidade do Estado do Amazonas.

Manaus, AM, Brasil.

2. Universidade Federal de Santa Catarina.

Florianópolis, SC, Brasil.
Autor Correspondente:

Darlisom Sousa Ferreira

E-mail: darlisom@uea.edu.br

\section{Recebido em 18/03/2020.}

Aprovado em 25/06/2020.

\begin{abstract}
Resumo
Objetivo: Descrever o processo de ideação e prototipagem de um aplicativo para dispositivo móvel de apoio à práxis educativa de enfermeiros da estratégia saúde da família. Método: Estudo metodológico com interface participativa e abordagem qualitativa, operacionalizado em quatro fases, segundo modelo adaptado do Design Participativo: Fase 1 - Exploração do contexto; Fase 2 - Ideação; Fase 3 - Prototipação; Fase 4 - Teste de usabilidade. Envolveu 26 enfermeiros de um distrito de saúde de Manaus, AM, Brasil. Dados coletados entre abril de 2018 e março de 2019, por meio da técnica World Café e formulário de teste de usabilidade, submetidos à análise de conteúdo categorial temática e estatística descritiva. Resultados: Foi produzido o protótipo FracTeam ${ }^{\circledast}$ APS, apresentado por Wireframe para briefing do estado atual. Como tecnologia estratégica e não convencional aglutinou funcionalidades e áreas temáticas múltiplas em alinhamento com necessidades do usuário enfermeiro. Conclusão e implicações para a prática: o processo possibilitou o exercício da experiência colaborativa em prol das necessidades intelectuais de educação e trabalho a partir da inteligência coletiva dos trabalhadores. A ideação e prototipagem com processos participativos repercutirão no desenvolvimento de tecnologias para a Enfermagem e saúde.
\end{abstract}

Palavras-chave: Educação em Saúde; Enfermagem em Saúde Comunitária;

Invenções; Aplicativos Móveis; Informática em Enfermagem.

\section{Abstract}

Objective: To describe the process of ideation and prototyping of a mobile application supporting for the educational practices of family health nurses. Method: Methodological study and participatory research, with a qualitative approach, conducted in four steps, according to a model adapted from Participatory Design: Step 1 - Exploration of the context; Step 2 - Ideation; Step 3 - Prototyping; Step 4 - Usability Testing. The study involved 26 nurses of a health district in Manaus, AM, Brazil. Data was collected between April 2018 and March 2019, using the World Café method and a usability testing form. Data were submitted to thematic and categorical content analysis and descriptive statistics. Results: The FracTeam ${ }^{\circledast}$ APS prototype was produced, presented by Wireframe for a briefing on the current status. As a strategic and unconventional technology it brought together multiple functionalities and thematic areas in alignment with the needs of the nurse user. Conclusion and implications for practice: the process enabled a collaborative experience to support the intellectual needs of education and work according to the collective intelligence of workers. Ideation and prototyping based on participatory processes will affect the development of technologies for nursing and health practice.

Keywords: Health Education; Community Health Nursing; Inventions; Mobile Applications; Nursing Informatics.

\section{Resumen}

Objetivo: Describir el proceso de ideación y prototipado de una aplicación para dispositivos móviles que apoye la práctica educativa de los enfermeros de la estrategia de salud familiar. Metodología: Se trata de un estudio metodológico con interfaz participativa y enfoque cualitativo, operativizado en cuatro fases, según el modelo adaptado del Diseño Participativo: Fase 1 - Exploración del Contexto; Fase 2 - Ideación; Fase 3 - Prototipado; Fase 4 - Prueba de Usabilidad. Participaron 26 enfermeros de un distrito de salud de Manaos, Amazonas, Brasil. Los datos recogidos entre abril de 2018 y marzo de 2019, a través del formulario de la prueba de usabilidad y de la técnica del World Café, se sometieron a un análisis de contenido categórico temático y a otro de estadística descriptiva. Resultados: El prototipo FracTeam ${ }^{\varpi}$ APS fue producido, presentado por Wireframe para una sesión informativa sobre el estado actual. Como tecnología estratégica y poco convencional, reunió múltiples funcionalidades y áreas temáticas en alineación con las necesidades del usuario de enfermería. Conclusión e implicaciones para la práctica: El proceso posibilitó el ejercicio de la experiencia colaborativa a favor de las necesidades intelectuales de la educación y el trabajo a partir de la inteligencia colectiva de los trabajadores. La ideación y el prototipado de los procesos participativos impactará en el desarrollo de tecnologías para la práctica de la Enfermería y la salud.

Palabras clave: Educación en Salud; Enfermería en Salud Comunitaria; Invenciones; Aplicaciones Móviles; Informática de Enfermería. 


\section{INTRODUÇÃO}

A inovação tecnológica é definida como processo de concepção ou de agregação de novas funcionalidades ou características de um produto ou de um método de produção. ${ }^{1}$ A inovação também é entendida como uma nova mentalidade, desencadeada por mudanças contextuais e que permite vínculos novos, úteis, criativos e inesperados, gerando uma solução/adaptação a um problema e agregando valor, seja no campo cientifico, acadêmico ou da prática.

Até a década passada a Enfermagem expressava uma incipiente atividade científica de inovação e desenvolvimento tecnológico, com frágil desempenho na geração de inventos. ${ }^{2}$ A demarcação e o desenvolvimento histórico da dimensão tecnológica na enfermagem se deram a partir da primeira metade do século XX na atuação profissional em saúde pública e estruturaram-se em diferentes períodos de construção, expressos nas seguintes fases: 1 - compreensão dos tempos de oscilação político-sociais e de qualidade da produção, 2- a fase de inventos e adaptações de enfermagem, e 3- o desenvolvimento de projetos que possibilitaram registros e patentes a partir da expansão dos cursos de graduação e pós-graduação. ${ }^{3}$

$\mathrm{Na}$ Enfermagem as inovações criam melhoria na qualidade, custo-efetividade ou eficiência. ${ }^{4}$ Nesse aspecto, destaca-se que a área tem evoluído na constituição de redes de conhecimento para construção de produtos oriundos de sua práxis e com isso vêm alcançando impacto significativo na qualidade do cuidado e melhoria da saúde da população. Tal investimento tem como alvo o incremento da inovação na atenção à saúde, à adequação às necessidades dos usuários como um todo, e na solução de problemas estruturais e globais da humanidade. .,5-7 $^{2,5}$

Uma tipologia das inovações em Enfermagem abarca 8 tipos, de acordo com os impactos esperados e os contextos de aplicação: 1) criar modelos de oferta de cuidados - ou atendimento; 2) transformar processos para melhorar a oferta de cuidados ou atendimento; 3 ) desenvolver intervenções de assistência ao paciente; 4) pesquisa avançada e métodos de tradução; 5) facilitar a comunicação e colaborações; 6) aproveitar a tecnologia e os dados; 7) habilitar transições de função; e 8) desenvolver o ensino de métodos. ${ }^{4}$

Considerando a amplitude dos cenários de trabalho da Enfermagem no Brasil, acredita-se que tecnologias devem ser desenvolvidas para contextos e demandas específicas, a partir do conhecimento das experiências reais de trabalho. Uma importante eleição se focaliza na qualificação do trabalho da Enfermagem na Estratégia Saúde da Família (ESF), pelo importante espaço que a atuação de Enfermeiros nela ocupa. Além disso, o caráter organizativo e substitutivo da ESF faz frente ao modelo tradicional de assistência primária, como estratégia para produção de impacto no sistema de saúde, ampliando a acessibilidade ao sistema de saúde e incrementando as ações de prevenção e promoção da saúde. ${ }^{8}$

Considerando a necessidade do incremento de tecnologias de informação e comunicação no apoio aos enfermeiros das equipes da $E S F,{ }^{9}$ se assume a práxis educativa de enfermeiros da ESF como a atividade-alvo para o desenvolvimento de uma tecnologia do tipo aplicativo móvel (App).

Ainda que etimologicamente o termo práxis possua o sentido aproximado de prática, este manuscrito assume sua acepção mais ampla, polissêmica e processual. Aquela alicerçada na dialética, na reflexão, traduzida no movimento da ação para além da conduta, permeada pela busca do conhecimento teórico e sua aplicação na realidade. A práxis na qual o homem se constrói e se conhece, promovendo a transformação de si mesmo e do mundo a sua volta. A princípio, elege-se o termo práxis para designar a atividade humana e social manifestada na relação teoria e prática. ${ }^{10}$ Assim, a forma específica de práxis a ser abordada, é a práxis educativa concretizada em manifestações individuais e coletivas de enfermeiros que atuam na Estratégia Saúde da Família (ESF).

Pensar sobre inovação para a práxis educativa de enfermeiros da ESF significa, entre outros aspectos, produzir instrumentais para além de produtos físicos industrializados. Perpassa a assunção do processo participativo para o pensamento co-criativo e co-produtivo do trabalhador dos serviços de atenção à saúde, estrategicamente em prol da construção de conceitos e métodos de desenvolvimento tecnológico, pautado na troca, na multiplicidade de visões, na inteligência coletiva e no aprendizado.

Atualmente a grande maioria de aplicativos móveis usados em diferentes campos não é apresentada em artigos científicos e ainda poucos são produzidos em vinculo com estudos acadêmicos. Daí que o método mais eficaz de localização deste tipo de produto tecnológico é por busca direta em lojas virtuais pra Android e iOS (Play Store e Apple Store), então ao realizar uma busca por app móveis relacionados à práxis educativa de enfermeiros na ESF, nessas lojas virtuais, foi encontrada a época do estudo apenas uma produção nacional, a Rede Humaniza SUS Móvel (RHS), uma rede social de pessoas envolvidas nos processos de humanização da gestão e do cuidado no SUS. Nos demais aplicativos disponíveis predominam as finalidades de treino, corpo e estética, nutrição, e dietoterapia na perspectiva do usuário. Para o trabalhador destaca-se às alternativas de formação na modalidade à distância e/ou TV play com pouca ou quase nenhuma interatividade.

Este estudo justifica-se, pois contribui tecnologicamente para o processo de trabalho em saúde na ESF a partir do protagonismo dos profissionais no desenvolvimento de um instrumental emergente da prática social na produção da saúde.

A relevância do estudo se dá por dois horizontes, o primeiro científico-imediato, sistematizando contribuições à práxis crítico-criativa na ESF; e o segundo científico-mediato, por criar possibilidades à expansão de novos/outros estudos participativos sobre trabalho, inovação tecnológica, educação em saúde e cidadania na região norte do país.

Considerando a pertinência do incremento da inovação tecnológica para o trabalho da Enfermagem em suas diferentes necessidades e contextos de atuação, o objetivo do estudo é descrever o processo de ideação e prototipagem de um aplicativo 
para dispositivos móveis para mediar a práxis educativa de enfermeiros da estratégia saúde da família.

\section{METODO}

Trata-se de estudo metodológico com interface participativa e abordagem qualitativa, operacionalizado em quatro fases: Fase 1 - Exploração inicial do trabalho - neste estudo o contexto do trabalho educativo na ESF. Fase 2 - Processo de Descoberta - Ideação. Fase 3 - Prototipagem. Fase 4 - Teste de usabilidade, modelo adaptado do Design Participativo (DP) para empoderamento e inclusão digital. ${ }^{11,12} \mathrm{O}$ Design Participativo surgiu a mais de meio século e tem sua origem em território escandinavo entre os anos 70 e 80 , sendo utilizado com o objetivo de "capacitar trabalhadores e promover a democracia no local de trabalho"12. O principal fundamento dessa metodologia é envolver os usuários no processo de desenvolvimento, ou seja, diferente dos modelos de design que são pensados "para" alguém, o DP trata o design "com" o usuário. Entre as vantagens do DP, observa-se o engajamento dos usuários nos processos de design e a maior aceitação do produto, uma vez que se sentem também responsáveis pelos resultados. As 4 Fases foram realizadas de junho de 2018 a maio de 2019.

Na Fase 1 o pesquisador se reuniu com os trabalhadores para uma primeira aproximação e familiarização com o problema, apresentação de ideias e estabelecimento de prioridades. Participaram 26 enfermeiros da Estratégia Saúde da Família do Distrito de Saúde Leste (DISAL), um dos cinco Distritos vinculados a Secretaria Municipal de Saúde (SEMSA), de Manaus, AM, Brasil). Foram utilizados os seguintes critérios de inclusão: ser efetivo do quadro da SEMSA; ter atuação mínima de 02 anos na mesma UBSF; estar em plena atividade no período da coleta de dados. A sensibilização e o convite aos participantes ocorreram por meio do contato telefônico seguida de visitas as UBSF do DISAL.

Para a produção de dados utilizou-se a técnica grupal World Café, que busca promover a descoberta e a construção participativa de soluções conjuntas para problemas coletivos, por meio do encontro de pessoas em torno de conversações significativas e estratégicas. ${ }^{13}$

Foram realizados dois encontros em momentos distintos (World Café 1 e 2), em um espaço privativo, reservado para a atividade. A técnica foi coordenada pelo anfitrião-pesquisador. Nestes espaços de encontro e diálogo, as conversas favorecem explorar sentidos para os múltiplos contextos de vida e trabalho. ${ }^{13}$ A questão norteadora, dos dois encontros foi: o que as equipes da estratégia saúde da família podem criar para potencializar as práticas de educação em saúde? Foram fornecidos materiais de apoio, como toalhas de papel e canetas para anotações e registro livre de ideias. Os participantes dos encontros do World Café foram distribuídos em grupos de quatro por mesa, cabendo a um deles o papel de anfitrião de mesa. Os diálogos foram organizados em rodadas de 15 minutos, de modo que todos os participantes circulassem nas mesas e, progressivamente, trocassem ideais entre si. O retorno às mesas iniciais se dava na última rodada, quando ocorria a síntese das descobertas e preparo do compartilhamento em plenária, oportunidade de expressão do conhecimento coletivo. Em média a duração dos encontros foi de 03 horas. Para contribuir com a logística, registros, controle da frequência e outros aspectos de infraestrutura, a pesquisa contou com auxiliares de pesquisa devidamente treinados e os encontros foram filmados e fotografados.

Os dados obtidos foram transcritos, organizados e processados com auxílio do software Atlas.ti8 (Qualitative Research and Solutions) versão 8.3.20/2019, incluindo o conteúdo das toalhas de mesa e vídeos. Trata-se de um CAQDAS (Computer Assisted Qualitative Data Analysis Software) utilizado em investigações qualitativas em diversas áreas e distintas abordagens teórico-metodológicas. ${ }^{14} \mathrm{~A}$ análise dos dados dos dois encontros foi de conteúdo categorial-temática.

Na Fase 2, pesquisador, desenvolvedor e trabalhadores de forma interativa idealizaram os requisitos para o invento tecnológico. Participaram 12 enfermeiros (dentre os 26 enfermeiros que participaram da Fase 1). Nesta Fase realizou-se o World Café 3. No primeiro momento, o pesquisador apresentou a síntese dos World Café 1 e 2, as categorias que emergiram da análise, com destaque para a categoria indicativa de criação de tecnologia, e a figura geométrica de um dodecágono, representativa do Marco Conceitual para Práxis Educativa de Enfermeiros da ESF, construído a partir da síntese da Fase 1, e representado na forma de um fractal. No segundo momento o desenvolvedor apresentou algumas opões tecnológicas disponíveis no mercado. Por fim, no terceiro momento, o pesquisador lançou a questão-guia da discussão: a partir da síntese dos cafés 1 e 2 e das opções apresentadas, que tecnologia poderá potencializar a práxis educativa em saúde na ESF e o que deverá conter?

As possibilidades tecnológicas do segundo momento ilustraram o processo de definição do invento e sua prototipagem, e sobre isso, é importante descreve-las, pois cada uma trás consigo (em funcionalidades separadas), o desejo dos usuários tê-las congregadas em uma única opção tecnologia em favor da práxis. O Believ é uma plataforma de grupos; o Timerepublik um aplicativo que permite a troca e o compartilhamento de experiências e habilidades profissionais; o Slack permite a interação grupal a partir do envio e recebimento de textos, imagens, vozes vídeos; e o Medium é um aplicativo de News, que permite a troca de conteúdos e/ou matérias científicas e/ou jornalísticas.

$\mathrm{Na}$ Fase 3, o pesquisador e o desenvolvedor, com base nos requisitos discutidos e apresentados no World Café 3 , desenvolveram um protótipo de aplicativo. Os fluxos foram hospedados na plataforma Marvel com os respectivos Wireframes. Trata-se de uma versão primitiva, que consiste na representação esquemática e/ou diagramada das estruturas e divisões de um projeto tecnológico a partir da experiência do usuário. ${ }^{15}$

Na Fase 4, o pesquisador e os trabalhadores, em reunião agendada e realizada em uma unidade de saúde do DISAL, testaram o protótipo do aplicativo. Participaram 8 enfermeiros (dentre os 26 enfermeiros que participaram das Fases 1 e 2). Os enfermeiros foram submetidos ao teste de usabilidade 
por meio de simulação digital intuitiva do protótipo. Quando submetidos à simulação do protótipo os trabalhadores foram observados e procederam ao preenchimento do Formulário de Teste de Usabilidade no GoogleForms com 2 seções contendo 18 questões objetivas e 1 subjetiva. Usabilidade é um termo referente a uma amplitude de métodos que avaliam a interação do usuário com as interfaces de produtos e sistemas. Esse estudo assume o processo UX (User Experience), que trata o processo de design a partir da influência dos usuários para além das características funcionais e finais, mas no andamento até a versão final do invento. ${ }^{15,16} \mathrm{~A}$ análise dos dados do teste foi quantitativa, com base na estatística descritiva.

O estudo recebeu anuência institucional da SEMSA, foi submetido à Plataforma Brasil e obteve parecer consubstanciado de aprovação pelo Comitê de Ética em Pesquisa da Universidade do Estado do Amazonas (UEA) sob a CAAE 796719170.0000.5016 e parecer № 2.376 .273 em 10 de novembro de 2017. Foram assinados o Termo de Consentimento Livre e Esclarecido e o Termo de Autorização de Uso de Imagem e Voz, amparados pelos princípios éticos das Resoluções 466/2012, 510/2016 e 580/2018 do Conselho Nacional de Saúde (CNS), que regem pesquisas com serem humanos, ciências sociais e interesse estratégico com o Sistema Único de Saúde (SUS).

\section{RESULTADOS}

No que tange a Fase 1 (World Café 1 e 2), as conversações significativas revelaram cinco categorias; dentre estas, a que apontou para a perspectiva tecnológica foi a categoria: práxis criadora de tecnologias para o trabalho educativo na ESF, constituída a partir de três conjuntos discursivos: criação de espaços de troca, compartilhamento e diálogo; uso de tecnologias de informação e comunicação na práxis educativa; presença da universidade na educação permanente do trabalhador da ESF (Figura 1). Nesta categoria os conteúdos discursivos aglutinam pistas tecnológicas com funcionalidades que apontam para tecnologias disponíveis no mercado, produtos com interfaces fragmentadas que podem ser reunidas em uma única tecnologia móvel. Neste estudo, dá-se destaque às Fases 2, 3 e 4.

No que tange a Fase 2, a discussão que ocorreu entre os trabalhadores (World Café 3), suscitou o processo de definição dos requisitos para o invento. Constatou-se que os requisitos se pautavam nas necessidades cotidianas dos trabalhadores, com vistas a potencializar as práticas educativas. Verificou-se que uma das necessidades dos profissionais era organizar a práxis em torno das áreas temáticas, conforme diretrizes do Ministério da Saúde: saúde da mulher, saúde da criança, saúde do idoso, etc. Verificou-se também que desejavam interagir com os membros da equipe bem como com as demais equipes do DISAL, para compartilhar experiências, dúvidas e sugestões. Foi com base nessas ideias dos trabalhadores que se seguiu para a Fase seguinte.

No que tange a Fase 3 , o processo foi operacionalizado pelo desenvolvedor a partir de elaboração de Wireframes, um método para criar um protótipo usado em design de interface que sugere a estrutura de um sítio, web, app, entre outros; uma ilustração semelhante do layout que contempla os elementos fundamentais de uma tecnologia/invento.

O protótipo foi nomeado FracTeam ${ }^{\circledR}$ APS, em que Frac representa fractal, Team equipe da ESF, APS atenção primária a saúde. Trata-se de uma tecnologia estratégica e não convencional que aglutina funcionalidades de múltiplos aplicativos em um único produto. $\mathrm{O}$ objetivo principal do Wireframe é ser a ferramenta principal de alinhamento entre a necessidade e expectativa do usuário final do invento. De forma preliminar, a apresentação do Wireframe ao usuário, promove a análise crítica/construtiva

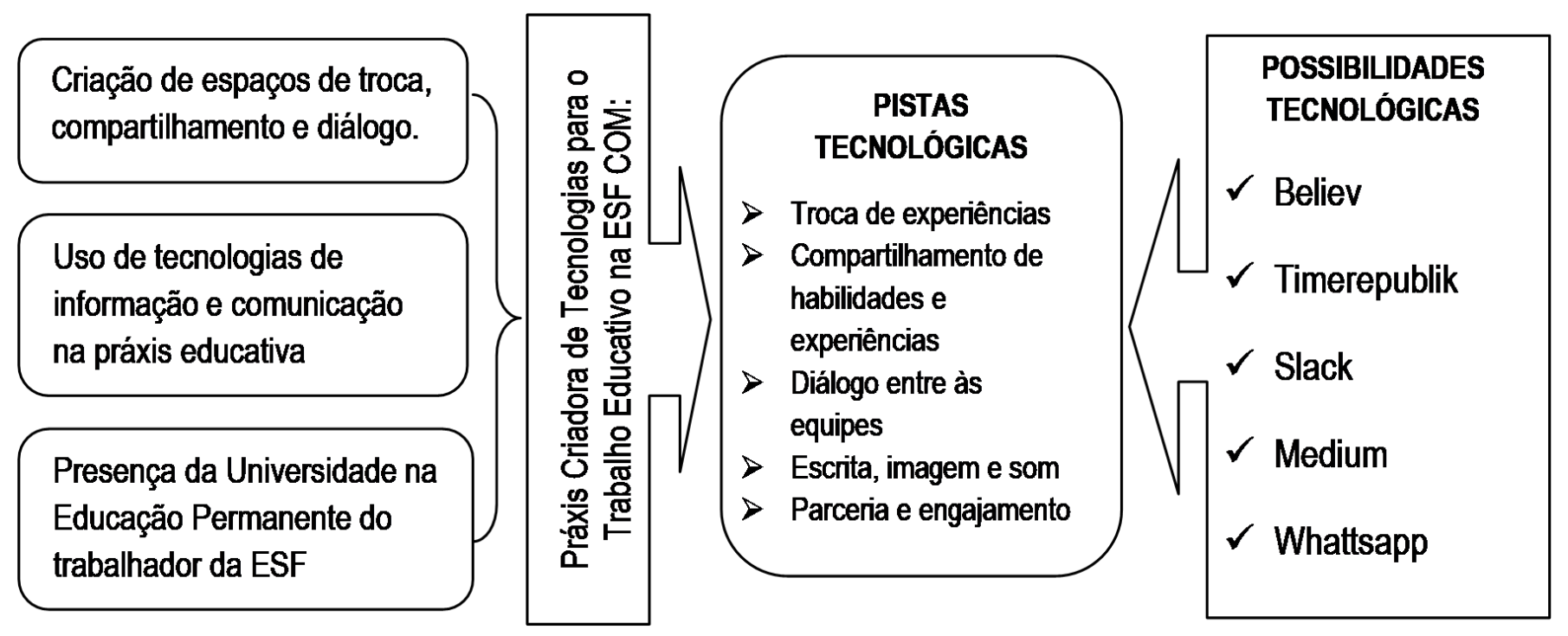

Figura 1. Síntese dos elementos constitutivos da categoria Práxis Criadora de Tecnologias para o Trabalho Educativo na ESF Fonte: os autores 
ao desenvolvimento acabado do produto, proporcionando a mensuração e/ou briefing do estado atual, com vistas à suficiência/aprovação e sucesso do produto (Figura 2).

O protótipo App FracTeam ${ }^{\circledR}$ é uma ferramenta digital projetado sob a perspectiva política de cognição dos trabalhadores da
ESF e o desejo pelo provimento de tecnologia "coorporativa pública" que oportunize o diálogo, a troca e o compartilhamento de conhecimentos e experiências em torno do trabalho educativo em saúde. Possui compatibilidade com smartphones e tablets que operam com tecnologia do tipo iOS ${ }^{\circledR}$ e Android $^{\circledR}$, e para
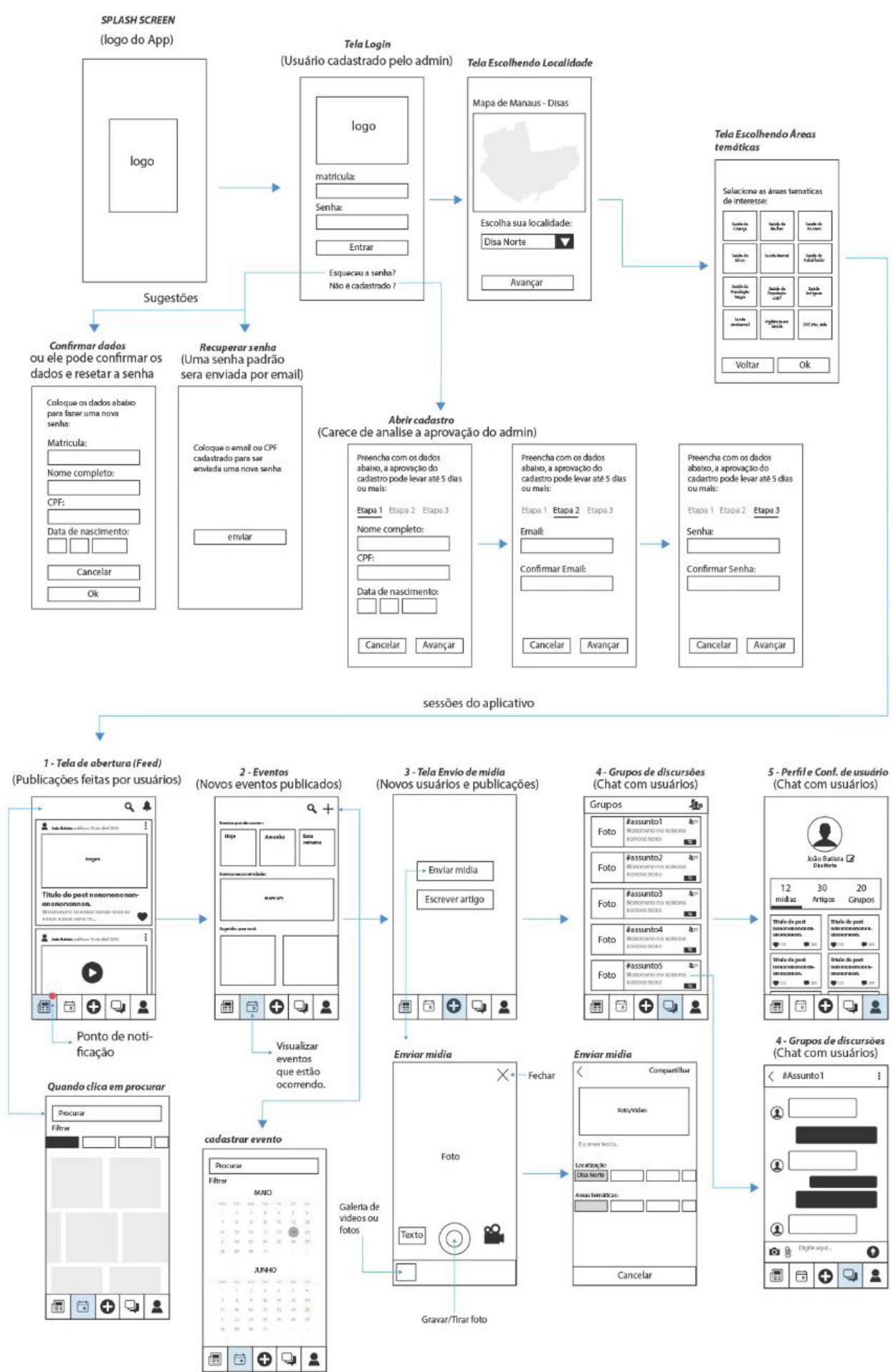

Figura 2. Prototipação - Wireframes do protótipo do App FracTeam ${ }^{\circledR}$ APS. 
efetuar o download, o usuário necessitará ser estar vinculado ao serviço de saúde e ter acesso à internet.

A tela inicial do protótipo exige matrícula funcional para que 0 enfermeiro realize o acesso com senha criada no primeiro acesso, a partir do banco de dados funcional previamente cadastrado, por tratar-se de tecnologia coorporativa aplicada ao ambiente funcional, sendo, portanto, de acesso restrito aos servidores do cenário da pesquisa. A segunda tela traz a escolha do Distrito de Saúde, no caso o Leste, com possibilidade de expansão para os demais distritos (Norte, Sul e Oeste), num possível desenho de aplicação/integralização a toda RAS. As telas seguintes contemplam aa áreas temáticas (Figura 3).

Ao selecionar a área temática o trabalhador acessa todas as funcionalidades do protótipo em cada área temática podendo cria eventos e grupos, enviar e receber conteúdos, vídeos, imagens; interagir com outros usuários e estabelecer híbridas formas comunicacionais, formativas e informativas.

No que tange a Fase 4 , os trabalhadores que participaram têm idade entre 31 e 57 anos, $88,9 \%$ usam Smartphones com sistema operacional Android e 11,1\% iOS. Quando perguntados sobre o horário de uso, 44,4\% informam acessar a qualquer hora do dia e $55,6 \%$ apenas a noite. No tangente às redes sociais $44,4 \%$ possui mais de 4 redes sociais instaladas no seu celular, 33,3\% duas, e 22,2\% apenas uma. Quando inquiridos sobre o acesso a internet no Distrito de Saúde, 66,7\% acessam frequentemente e $33,3 \%$ às vezes.

Sobre a experiência com o protótipo de App FracTeam ${ }^{\circledR}$, $55,5 \%$ considerou totalmente adequado, $33,3 \%$ adequado e $11,1 \%$ parcialmente adequado. Ao serem perguntados sobre a rapidez das ações do protótipo $55,6 \%$ considerou rápida e $44,4 \%$ moderada. Em relação às dificuldades para navegar, criar e enviar conteúdo, $66,7 \%$ não relatou dificuldades e 33,3\% teve parcial dificuldade.

Quanto às funções apropriadas, agradáveis e esperadas no protótipo, $100 \%$ dos sujeitos considerou totalmente satisfatória. Quando perguntados sobre sugestões e observações a cerca do protótipo os usuários relataram:

O aplicativo de maneira geral preenche as expectativas (Enf1. Informação verbal).

Estou extremamente satisfeita, é uma ferramenta de fácil manuseio e entendimento, muito semelhante a outros aplicativos existentes no mercado, acredito que será de grande proveito para ampliar diálogos, discussões e o conhecimento para os profissionais que atuam na Atenção Básica, isso um maior acesso e melhor qualidade no atendimento aos usuários (Enf2. Informação verbal).

É inovador, mas penso que deveria apresentar conteúdos em paralelo aos do Ministério da Saúde (Enf3. Informação verbal).

Acredito que este aplicativo oportunizará feedback positivo enquanto educação permanente e alinhamento de condutas e orientações aos profissionais da rede (Enf4. Informação verbal).

Inclusão de tabelas de CID10 e CIAP2 (Enf5. Informação verbal).
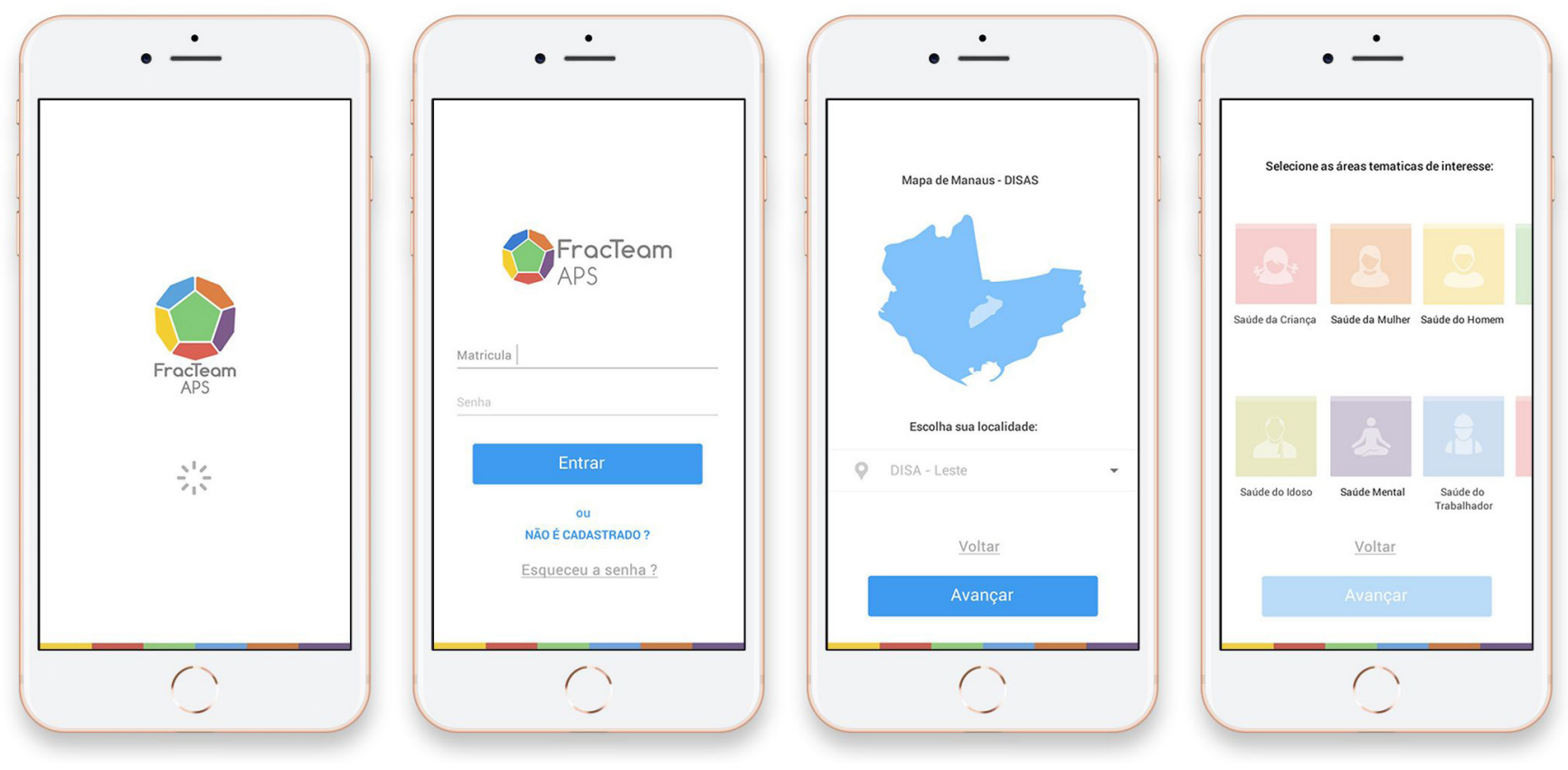

Figura 3. Tela Inicial, Acesso Funcional, DISA e Áreas Temáticas de Interesse - Protótipo do App FracTeam ${ }^{\circledR}$ APS. Fonte: os autores 


\section{DISCUSSÃO}

A utilização da técnica World Café com os trabalhadores participantes deste estudo promoveu o encontro de enfermeiros, e por meio de conversações significativas e estratégicas, buscaram refletir e discutir sobre o contexto em que trabalham e realizam a práxis educativa bem como discutiram e apontaram requisitos para alternativa tecnológica para potencializá-la.

A técnica possibilita que as conversas favorecem a descoberta e a construção participativa de soluções para problemas coletivos. É uma técnica que reaviva profundas lembranças em relação a duas crenças fundamentais sobre a vida humana: - queremos conversar em conjunto a respeito das coisas que são importantes para nós; - à medida que conversamos, nos tornamos capazes de acessar uma sabedoria maior àquela que se encontra apenas no coletivo. ${ }^{13}$

A aplicação do modelo de Design Participativo (DP), diferentemente do modelo de Design Centrado no Usuário (DCU), favoreceu a co-produção, pois os trabalhadores tiveram voz ativa na discussão de problemas, elaboração de ideias e indicativos para o design da tecnologia. ${ }^{15}$ Neste modelo, as oportunidades de expressão coletiva são favorecidas, além dos testes de usabilidade tradicionais e mais comuns.

Com a ação conjunta entre pesquisador, desenvolvedor e trabalhadores no processo, atingiu-se alta intensidade participativa no estudo metodológico desenvolvido. ${ }^{17} \mathrm{~A}$ alta intensidade é viabilizada com a co-criação. No desenvolvimento participativo de instrumentos-tecnologias, promove-se interação-diálogo, e as conversações viabilizam a identificação tanto de conteúdos como de aspectos de aparência, requisitos primordiais para uma prototipação.

Das conversações emergiram as pistas tecnológicas que suscitaram nos requisitos para o desenvolvimento do protótipo do tipo aplicativo. O desenvolvimento de aplicativos tem se tornado cada vez mais frequente na atualidade. Os processos informatizados são ferramentas que aperfeiçoam e simplificam as ações do enfermeiro, seja no campo da gerência, da assistência ou da docência, desta forma estando relacionados com o processo de trabalho deste profissional. ${ }^{18}$ Desse modo, o uso de evidências científicas e o envolvimento do usuário (neste estudo, os trabalhadores da ESF), respalda a forma como foi desenvolvido, o objetivo e uso para o qual o protótipo App FracTeam $^{\circledR}$ se destina. ${ }^{19}$

Os resultados indicaram que as necessidades dos trabalhadores poderiam ser atendidas por meio de um recurso tecnológico. Quando convidados a pensar sobre uma tecnologia para potencializar a práxis educativa, foram geradas ideias para apoio ao trabalho realizado. Idear implica gerar, inaugurar, inventar. Caracteriza-se pelo encadeamento de ideias para concepção/formação de um intento ${ }^{20}$ ou uma técnica para inovação e avanço do conhecimento em torno de uma situação que permite a quebra do status quo. Conceber, projetar e desenvolver recursos tecnológicos de apoio ao trabalho por meio de interfaces digitais traduz-se uma demanda de desafio crescente, que requer método científico, participativo e inclusivo por parte de pesquisadores, designer e desenvolvedores. Neste sentido, a inovação tecnológica idealizada e prototipada se inscreve de forma muito oportuna, ao aliar o moderno e relevante, à adequada fundamentação conceitual a prática em curso e aos instrumentais necessários à concretização da práxis. ${ }^{20}$

O protótipo produzido com os trabalhadores se inscreve enquanto inovação tecnológica, tecnologia de autoeducação e educação em rede, com potencial de transformação da práxis educativa com base no encontro entre o "mundo do trabalho" e o "mundo do usuário" e na interseção entre "o ensinar saúde" e o "praticar saúde" na realidade dos territórios da ESF.11,12,15

O App FracTeam ${ }^{\circledR}$ constitui-se alternativa de tangibilização de problemas e apoio à práxis educativa de enfermeiros da Estratégia Saúde da Família. Trata-se de um protótipo de tecnologia de comunicação, informação (e formação), que contempla aspectos a partir da experiência dos usuários e suas necessidades tecnológicas. Existe a previsão de uso do App no cenário de estudo, não apenas no distrito e unidades de saúde estudadas, mas por toda a Secretaria de Saúde do Município de Manaus, após as fases finais do estudo. Além disso, pretende-se avançar na participação no componente moderação e nas formas de participação da gestão dentro do App, além da possibilidade de disponibilizá-lo gratuitamente à todas as equipes de Manaus e dos mais diferentes cenários de ESF do país.

A inovação tecnológica causa impacto na vida de seus usuários, provoca transformações na vida (e trabalho). Não se trata simplesmente de lançar um "novo produto", mas de envolver o ser humano na concepção teórica e no desenvolvimento tecnológico dessa solução, propondo, testando, construindo e ajustando. É preciso, no entanto, agregar à proposição de uma tecnologia inovadora, o compromisso e apoio institucional para implementação do produto em uma etapa seguinte do programa. ${ }^{21}$

As ações de educação em saúde e o gerenciamento do cuidado conseguem ser desenvolvidos e fortalecidos pelo uso das tecnologias, ${ }^{20}$ já que a disseminação das informações ocorre com consistentes bases científicas, porém de forma atrativa. Podendo contribuir com a interatividade e inclusão tecnológica dos trabalhadores e o conhecimento necessário à redução de riscos relacionados ao cuidado em saúde da população.

Também na educação em Enfermagem vem sendo estudado os benefícios e barreiras do uso da tecnologia móvel no ensino clínico de enfermagem. Professores de enfermagem são desafiados a co-projetar e avaliar aplicativos móveis para apoiar o aprendizado na prática profissional, além de pesquisar os resultados de tal uso, as melhores estratégias de personalizar e integrar os apps no contexto educacional. ${ }^{22}$

A atual fase do desenvolvimento de inovações tecnológicas na área de informática em Enfermagem sugere a necessidade de conhecer e avaliar as decisões que integram o processo de desenvolvimento e seus contextos políticos. No futuro, a área mudará seu foco do desenvolvimento de marcas e dispositivos específicos aplicados à clínica para enfatizar o envolvimento e a autonomia do próprio usuário do cuidado, além de maximizar a usabilidade e interoperabilidade entre vários dispositivos e investimentos. ${ }^{23}$ 
No caso de tecnologias voltadas para promover e qualificar a comunicação entre profissionais o envolvimento dos usuários pode se dar desde as etapas de desenvolvimento. Ainda mais especificamente, no caso do tipo de tecnologia proposto neste estudo caberá desafios para o futuro, dentre estes, ampliar seu potencial de interoperabilidade, incorporando o diálogo com outros recursos tecnológicos.

A prototipagem ganha relevância e diminui a possibilidade do insucesso de um invento. A cultura da co-criação empática (ou empatia co-criativa) quando exercida no processo de desenvolvimento tecnológico, gera um "produto empático", acelera a velocidade de adaptação bem como a compreensão da importância do seu uso. ${ }^{15}$

Os resultados assinalaram que os testes de usabilidade podem indicar aperfeiçoamentos nos dispositivos desenvolvidos ou em prototipações como neste estudo, bem como indicar a aceitabilidade e o nível de satisfação dos usuários, aspectos considerados primordiais em estudos de usabilidade. ${ }^{24}$

\section{CONCLUSÃO E IMPLICAÇÕES PARA A PRÁTICA}

O processo possibilita o exercício da experiência colaborativa em prol das necessidades intelectuais de educação e trabalho a partir da inteligência coletiva. A ideação e prototipagem a partir de processos participativos repercutirão no desenvolvimento de tecnologias para a prática de Enfermagem e saúde. O App FracTeam ${ }^{\circledast}$ poderá fomentar o processo de aprendizagem, autoeducação e senso crítico do enfermeiro da APS, oportunizando, a partir do uso de dispositivos móveis pautados na práxis, o (re) direcionamento das ações pelo conhecimento colaborativo, contextual e experiências coletivas.

Um recurso tecnológico poderá promover a aquisição de conhecimentos, (re)aplicação de experiências exitosas, respaldando o planejamento e o cuidado na atenção à saúde das comunidades e territórios da ESF com potencial transformador dos mecanismos tradicionais de educação no trabalho. $O$ uso e expansão dos aplicativos móveis no processo de trabalho em saúde, enfermagem e educação requer como base um marco conceitual com fundamentos que alicerçam o seu desenvolvimento consistente e coerente com o real. Todo produto deve (ou deveria) partir do conhecimento crítico focado na vida e no ser humano. Inovar a partir do saber previamente conceptualizado é condição para o desenvolvimento tecnológico.

Se consideram como limitações do estudo a não escuta dos gestores, a fim de obter outras perspectivas sobre os desafios da práxis educativa dos enfermeiros da ESF, e a não inclusão dos enfermeiros da ESF lotados nos demais distritos de saúde do município.

Destaca-se o potencial para estudos futuros, em que o aplicativo seja apreciado e avaliado por especialistas bem como desenvolvido e testado com os enfermeiros da ESF. Recomenda-se que outras pesquisas sejam realizadas considerando o ponto de vista dos demais profissionais da ESF.

\section{AGRADECIMENTOS}

Ao Curso de Doutorado Interinstitucional entre Universidade Federal de Santa Catarina e Universidade do Estado do Amazonas. A Secretaria Municipal de Saúde e Escola de Saúde Pública de Manaus e aos Enfermeiros(as) da Estratégia Saúde da Família do Distrito Leste de Saúde de Manaus.

\section{CONTRIBUIÇÕES DOS AUTORES}

Darlisom Sousa Ferreira: Desenho do estudo. Aquisição, análise de dados e interpretação dos resultados; Redação e revisão crítica do manuscrito; Aprovação da versão final do artigo; Responsabilidade por todos os aspectos do conteúdo e a integridade do artigo publicado.

Flávia Regina Souza Ramos: Desenho do estudo. Aquisição, análise de dados e interpretação dos resultados; Redação e revisão crítica do manuscrito; Aprovação da versão final do artigo; Responsabilidade por todos os aspectos do conteúdo e a integridade do artigo publicado.

Elizabeth Teixeira: Desenho do estudo. Aquisição, análise de dados e interpretação dos resultados; Redação e revisão crítica do manuscrito; Aprovação da versão final do artigo; Responsabilidade por todos os aspectos do conteúdo e a integridade do artigo publicado.

\section{EDITOR ASSOCIADO}

Candida Caniçali Primo

\section{REFERÊNCIAS}

1. Marziale MHP. Pesquisa estratégica, inovação tecnológica e enfermagem Rev Latinoam Enferm. 2010 fev;18(1):1-2. http://dx.doi.org/10.1590/ S0104-11692010000100001. PMid:20428689.

2. Coelho MJ.Produtos de cuidados de enfermagem. Rev Bras Enferm. 2009 dez;62(6):912-22. http://dx.doi.org/10.1590/S0034-71672009000600020. PMid:20098887.

3. Paim L, Martins CR, Paese F, Bresciani HR, Callegaro GD. Demarcação histórica da enfermagem na dimensão tecnológica. Texto Contexto Enferm. 2009 set;18(3):542-8. http://dx.doi.org/10.1590/S010407072009000300018.

4. Joseph M, Huber DL, Bair H, Moorhead S, Hanrahan K. A typology of innovations in nursing. J Nurs Adm. 2019 jul/ago;49(7-8):389-95. http:// dx.doi.org/10.1097/NNA.0000000000000773. PMid:31335521.

5. Lorenzini Erdmann A. A inovação em enfermagem. Cienc Enferm. 2013;19(3):7-9. http://dx.doi.org/10.4067/S0717-95532013000300001.

6. Lorenzetti J, Trindade LL, Pires DEP, Ramos FRS. Tecnologia, inovação tecnológica e saúde: uma reflexão necessária. Texto Contexto Enferm. 2012 jun;21(2):432-9. http://dx.doi.org/10.1590/S0104-07072012000200023.

7. Barra DCD, Nascimento ERP, Martins JJ, Albuquerque GL, Erdmann AL. Evolução histórica e impacto da tecnologia na área da saúde e da enfermagem. Rev Eletr Enf. 2006 abr/jun;8(3):422-30.

8. Pinto LF, Giovanella L. Do Programa à Estratégia Saúde da Família: expansão do acesso e redução das internações por condições sensiveis à atenção básica (ICSAB). Cien Saude Colet. 2018 jun;23(6):1903-14. http://dx.doi.org/10.1590/1413-81232018236.05592018. PMid:29972498.

9. Pinto LF, Rocha CMF. Inovações na Atenção Primária em Saúde: o uso de ferramentas de tecnologia de comunicação e informação para apoio à gestão local. Cien Saude Colet. 2016 maio;21(5):1433-48. http:// dx.doi.org/10.1590/1413-81232015215.26662015. PMid:27166893. 
10. Pereira DA, Rocha SFM, Chaves PM. O conceito de práxis e a formação docente como ciência da educação. Rev Ciênc Hum. 2016 dez;17(29):31-45.

11. Bonfim CJL, Mombach JG, Martins ABN, Sousa JKL. Design Participativo: uma experiência de criação de aplicativos com meninas. Rev Sistemas e Comunicação [Internet]. 2018 jul./dez; [citado 2017 maio 29];8(2):40217. Disponível em: https://revistas.unifacs.br/index.php/rsc/article/ view/5780/3669

12. Spinuzzi C. The methodology of participatory design. Technical communication [Internet]. 2005 maio; [citado 2017 maio 29]; 52(2):163-74. Disponível em: https://www.researchgate.net/publication/233564945_ The_Methodology_of_Participatory_Design

13. Brown J, David I. O World Café: Dando forma ao nosso futuro por meio de conversações significativas e estratégicas. São Paulo: Cultrix;2007.

14. Prado ML, Souza ML, Monticelli M, Cometto MC, Gómez PF. Investigación cualitativa en enfermería: metodología y didáctica. 1. ed. Washington (DC): Organización Panamericana de la Salud; 2013.

15. Grilo A. Experiência do usuário em interfaces digitais: compreendendo o design nas tecnologias de informação [Internet]. Natal: SEDIS-UFRN 2019 [citado 2018 dez 05]. Disponível em: https://repositorio.ufrn.br/ jspui/handle/123456789/27011

16. Oliveira M, Seabra RD, Mattedi AP. Usabilidade de aplicativos de segurança colaborativa para smartphones: uma revisão sistemática. Rev Sistemas e Computação [Internet]. 2018 [citado 2018 dez 05] 8(2):262-76. Disponível em: https://revistas.unifacs.br/index.php/rsc/ article/view/5581

17. Teixeira E. Interfaces participativas na pesquisa metodológica para as investigações em enfermagem. Rev Enferm UFSM. 2019; 9:1-3. https:// doi.org/10.5902/2179769236334.
18. Juliani CMCM, Silva MC, Bueno GH. Avanços da informática em enfermagem no Brasil: revisão integrativa. J Health Inform [Internet] 2014 out/dez; [citado 2019 ago 9]; 6(4):161-5. Disponível em: http:// jhi-sbis.saude.ws/ojs-jhi/index.php/jhi-sbis/article/view/322

19. Curtis KE, Lahiri S, Brown KE. Targeting parents for childhood weight management: Development of a theory-driven and user-centered healthy eating app. JMIR Mhealth Uhealth. [Internet]. 2015 abr/jun; [citado 2019 ago 9]; 3(2):1-18. Disponível em: https://mhealth.jmir.org/2015/2/e69/

20. Ventola CL. Mobile devices and apps for health care professionals: Uses and benefits. J clin pharm ther. [Internet]. 2014 maio; [citado 2019 ago 9]; 39(5):356-64. Disponível em: https://www.ncbi.nlm.nih.gov/pmc/ articles/PMC4029126/

21. Ilha S, Santos SSC, Backes DS, Barros EJL, Pelzer MT, Oliveira AMN (Geronto) Tecnologia cuidativo-educacional na doença de Alzheimer e no apoio ao idoso/família: perspectiva dos docentes e discentes. Esc Anna Nery Rev Enferm. 2017 fev;21(2):1-8. http://dx.doi.org//10.5935/14148145.20170039

22. O'Connor S, Andrews T. Smartphones and mobile applications (apps) in clinical nursing education: A student perspective. Nurse Educ Today. 2018 out;69:172-8. http://dx.doi.org/10.1016/j.nedt.2018.07.013. PMid:30096510.

23. Wang J, Gephart SM, Mallow J, Bakken S. Models of collaboration and dissemination for nursing informatics innovations in the 21st Century. Nurs Outlook. 2019 jul/ago;67(4):419-32. http://dx.doi.org/10.1016/j. outlook.2019.02.003. PMid:30876686.

24. Weegen S, Verwey R, Tange HJ, Spreeuwenberg MD, Witte LP. Usability testing of a monitoring and feedback tool to stimulate physical activity. Patient Prefer Adherence [Internet]. 2014 mar;[citado 2018 jul 24] 8:311-22. Disponível em: http://www.ncbi.nlm.nih.gov/pmc/articles/ PMC3962312/?tool=pubmed

\footnotetext{
a Recorte da tese de doutorado "PRÁXIS EDUCATIVA DE ENFERMEIROS NA ESTRATÉGIA SAÚDE DA FAMÍLIA: do real conceptualizado à inovação tecnológica", de autoria de: Darlisom Sousa Ferreira, sob a orientação de Flávia Regina Souza Ramos. Programa de Pós-graduação em Enfermagem. Universidade Federal de Santa Catarina. Defesa em 30/07/2020.
} 